\title{
Food insecurity may be linked to childhood obesity in low-income Mexican-American families
}

by Patricia B. Crawford, Cathi L. Lamp, Yvonne Nicholson, Sarah Krathwohl, Mark Hudes and Marilyn S. Townsend

The purpose of this study was to examine the associations between past and current maternal food insecurity and child-feeding practices among low-income MexicanAmerican families. Participants in the study were mother-child pairs enrolled in the Special Supplemental Nutrition Program for Women, Infants and Children (WIC). The findings suggest that low-income MexicanAmerican mothers who are currently experiencing food insecurity were more likely to worry that their children were eating too much food and tended to offer smaller portion sizes to their children than mothers not currently experiencing food insecurity. Mothers who were overweight were more than twice as likely to have overweight children than mothers who were not overweight.

W hile excess weight is a concern across nearly all age levels in the United States, the emergence of pediatric overweight at earlier childhood ages is of particular concern due to its association with type 2 diabetes and chronic disease risk. Among children from 2 to 5 years old, the prevalence of overweight rose from $7 \%$ to $10 \%$ over the last decade (NCHS 2004). Moreover, there are ethnic disparities in the prevalence of childhood overweight (Crawford et al. 2001). In this age range, Mexican-American children were more likely to be overweight than those who were non-Hispanic black or nonHispanic white (13\% versus 9\%, respectively) (NCHS 2004).

Rates of excess weight and obesity are also higher within low-income

populations. Paradoxically, this is the same socio-demographic group that is affected by food insecurity (Crawford, Townsend, et al. 2004). The prevalence of food insecurity is highest for Hispanic and African-American households $(22 \%)$ and households living below the federal poverty level (38\%). In 2002 , twice as many households with children under 18 years old experienced food insecurity compared to households without children (17\% versus $8 \%$, respectively) (Nord 2003). Recent research suggests that food insecurity may play a role in the onset of obesity among some low-income groups (Olson 1999; Kendall et al. 1996).

\section{Child-feeding practices}

Assuming that parental childfeeding practices are related to childhood overweight, interventions can focus on modifying these practices in order to prevent obesity in children.

Food restriction. Involuntary food restriction may result in preoccupation with food, overeating and/or disor- dered eating, and weight gain (Kendall et al. 1996; Townsend et al. 2001). Studies suggest that food deprivation in childhood may induce binge eating behavior and overeating when food is plentiful (Fisher and Birch 1999). Nutrition educators have reported that overeating when food is available is a common food practice for participants in the Expanded Food and Nutrition Education Program (EFNEP) and Food Stamp Nutrition Education (FSNE) program (Kempson et al. 2002).

It is also possible that women who have experienced past food insecurity may practice restrictive feeding in an attempt to accommodate anticipated food shortages. However, this practice may disrupt natural systems of selfregulation. Limiting foods may promote the child's desire for certain foods, causing disregulation of caloric intake, overeating and ultimately excess weight gain. There is consistent data indicating that in noncontrolling, noncoercive households where children have access to a wide variety of healthy foods, chil- 

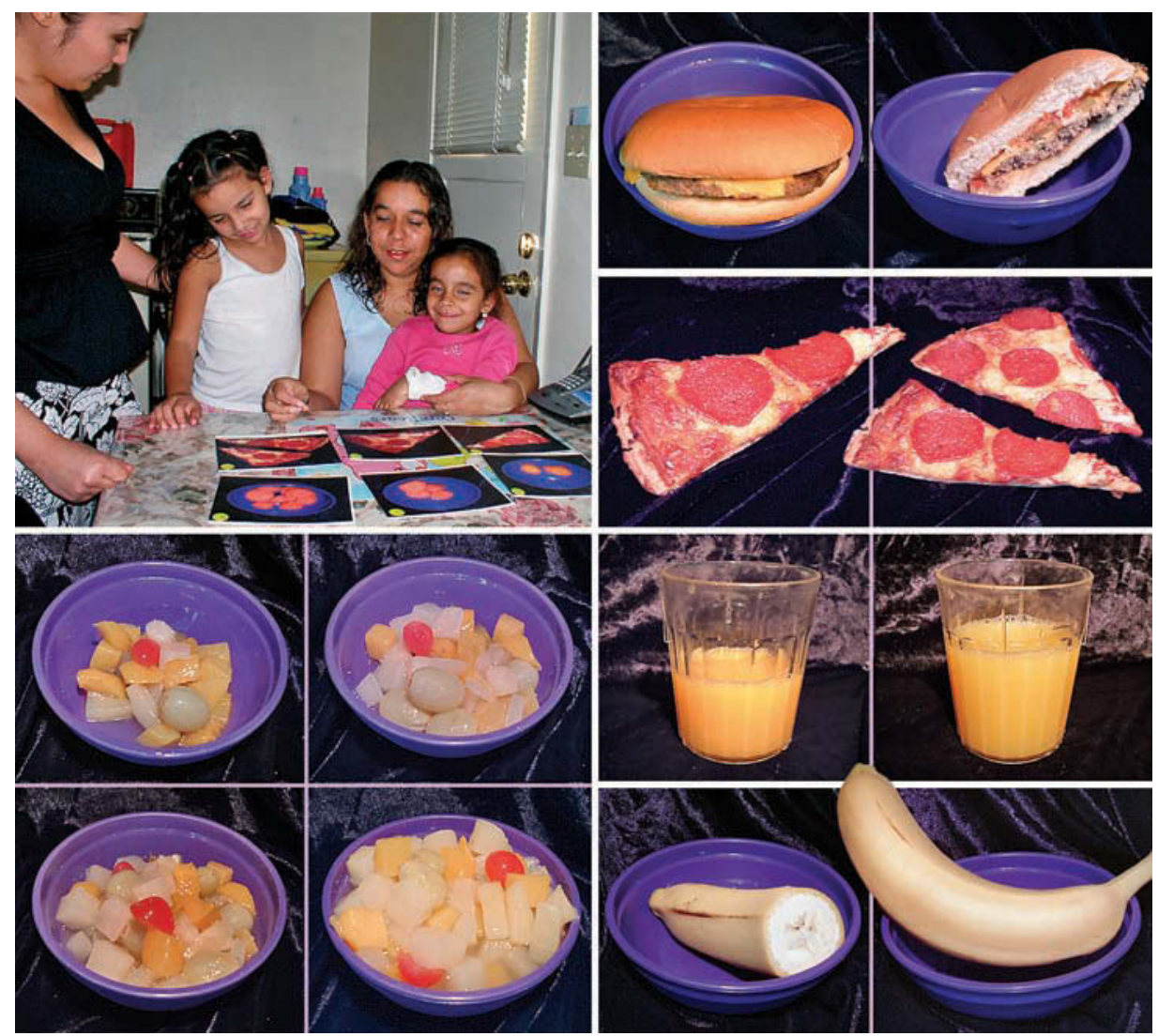

Mothers were shown pictures of different portion sizes for a variety of foods, in order to assess the typical amounts served to their children. Mothers currently experiencing food insecurity generally offered smaller portions of certain foods, such as French fries and chicken nuggets.

dren have the ability to self-regulate the amount of food and energy consumed (Johnson 2000).

Emotional feeding. Children's overeating may be associated with the childfeeding practice known as "emotional feeding"; for example, a parent may give a child something to eat to make him feel better or may use food as a reward. Food may be an index of caring attention in Mexican families, with parents indulging their children through sweet treats and snack foods (Brewis 2003).

Portion sizes. Increasing portion sizes have paralleled obesity increases over the last 3 decades. There is evidence that portion sizes are increasing even for children as young as 1 to 2 years old (McConahy et al. 2002). Studies have shown that more calories are consumed when larger portion sizes are offered (Engell et al. 1995; Rolls et al. 2002). Examining experiences of food deprivation and insecurity may be informative to understanding perceptions regarding the appropriateness of portion sizes.

\section{Food insecurity, child-feeding study}

To examine these issues, a study was designed to assess the impact of maternal food insecurity, both past and current, on child-feeding practices that are associated with weight gain among children in low-income families enrolled in the Special Supplemental Nutrition Program for Women, Infants and Children (WIC). Three main research questions guided the study. First, are mothers who are currently experiencing food insecurity or who have experienced food insecurity in their past more likely than other mothers to: (A) be indulgent with respect to feeding their child, (B) control or restrict their children's food, $(C)$ use food as a reward or (D) offer their children larger portion sizes? Second, are mothers who are overweight or obese themselves more likely to engage in these child-feeding practices? Third, are mothers who engage in these childfeeding practices more likely to have children who are overweight?

\section{Glossary}

Adiposity: Body fatness, specifically the body-fat tissue.

Food insecurity: Limited or uncertain availability of a safe and nutritionally adequate food supply.

Food security: Access to a relatively stable, safe and nutritionally adequate food supply.

Overweight and obesity (adults): Excess body weight as defined using height and weight indicators. Adults with a body mass index (BMI) over $25\left(\mathrm{~kg} / \mathrm{m}^{2}\right)$ are considered overweight; those with a BMI over 30 are defined as obese.

Overweight (children): For children, overweight status is defined using sex- and age-specific growth charts developed by the Centers for Disease Control and Prevention, with "at risk" for overweight being defined as a BMI between the 85th and 95th percentile, and overweight being defined as a BMI that is more than the 95th percentile.

Participants. Subjects were recruited from WIC participants in two California counties: Tulare, which is in the Central Valley and is rural, and Sacramento, which is urban. WICeligible Latino mothers of children 3 to 5 years old were invited to participate at the time of their regularly scheduled WIC visit. All study participants signed an informed consent form. Participants were told that their participation or nonparticipation would have no effect on their WIC enrollment or benefits. As an incentive, each participant received a \$10 gift card from Save Mart or Target.

Working in collaboration with WIC, two bilingual Cooperative Extension staff members interviewed each participant. Interviews were conducted in person, in Spanish or English, at the local WIC office or community multiservice health clinic. Anthropometric data and questionnaires concerning feeding practices and food insecurity were collected and analyzed for 74 mother-child pairs. We also collected the questionnaires but not anthropo- 
metric data for an additional 49 motherchild pairs, for a total of 123 pairs of mothers and children.

Data collected. We used six measurement tools for this study.

(1) The participant screener was used to identify and exclude mothers who were pregnant or postpartum, or who had children with physical disabilities.

(2) The demographic enrollment form captured basic information including the mother's date of birth, birthplace and childhood home, language spoken in the home, level of education and household income.

(3) The Child Feeding Practices Questionnaire included 21 questions related to restriction/maternal control, indulgence, food as a reward and other child-feeding behaviors. This instrument was adapted from one developed and validated by Birch et al. (2001). Questions related to restriction/maternal control included: "Do you scold your child for not eating well?" and "I have to be sure that my child does not eat too many sweets." Questions related to indulgence in child feeding included: "If your child does not want to eat at mealtime, do you offer other foods?" and "Does your child take food from the refrigerator, or a shelf, for a snack whenever she/he is hungry between meals?" Questions related to using food as a reward included: "I offer my child his/her favorite foods in exchange for good behavior" and "How often do you give your child food to keep him/her quiet?"

Questions were added to assess the portion sizes of specific foods that mothers serve to their children. This tool included 17 sets of photographs of foods in bowls, to provide a physical reference for portion size. Each set contained two to six photographs of varying portion sizes of a particular food. For each food, the range of portion sizes was developed based on the daily serving size recommended for 2 to 6 year olds in the USDA Food Guide Pyramid for Young Children (USDA 1999). The food portions portrayed were ranked on a four-point scale. Based on these photographs, participants were asked to choose the serving size that most accurately corresponded to the amount they feed their child. The corresponding weight of the selected portion size was then recorded in grams. This tool was validated by comparing subjects' responses to their child's food intake, as measured in a 24-hour recall. A nominal correlation was found between mother's preferences for offering larger serving sizes and child's total energy intake $(\mathrm{r}=0.18, P=0.1068)$.

(4) The USDA Food Security/Hunger Core Module Questionnaire (Bickel et al. 2000) was administered to assess current food insecurity in households. This instrument includes screener questions and an 18-item food-security measurement scale.

(5) The Past Food Insecurity

Questionnaire, which was developed by this research team (Crawford, with an electronic scale. Shoes and outer clothing were removed for the height and weight measurements.

For mothers, a BMI between 25 and 30 was defined as being overweight, and a BMI of 30 or more was defined as obese. For children, those with a BMI greater than or equal to the 95th percentile using sex- and age-specific growth charts developed by the Centers for Disease Control and Prevention were categorized as overweight. Children with BMIs between the 85th and 95th percentile were classified as "at risk for overweight." The advantages of using BMI to measure adiposity include its reliability, noninvasiveness, affordability and ease of calculation.

Prior to data collection, UC Cooperative Extension staff from Sacramento and Tulare counties attended a training and certification

\section{Fewer than a third of the mothers with overweight children perceived their child as being overweight or heavier than children the same age.}

Townsend, et al. 2004), includes 10 dichotomized (yes/no) questions about the level of food security the mother experienced as a child. The construct validity of this instrument was determined based on correlations with the size of the mother's childhood household and past food insufficiency. A household is considered food insufficient if household members sometimes or often do not have enough to eat (Ribar and Hamrick 2003). Past food insecurity was strongly correlated with household size $(r=0.31$, $P<0.003)$ and food insufficiency $(\mathrm{r}=$ $0.70, P<0.0001)$, which were the comparative measures for validation.

(6) Height and weight measurements were assessed for both mothers and children. This data was used to calculate body mass index (BMI), which is defined as body weight in kilograms divided by the square of height in meters $\left(\mathrm{kg} / \mathrm{m}^{2}\right)$. Height was measured to the nearest 0.1 centimeter using a portable stadiometer with a fitted headpiece. Body weight was measured to the nearest 0.1 kilogram program to standardize data collection procedures across the two study sites. In accordance with study protocols, one of the two bilingual data collectors administered the data collection instruments to each mother during an interview lasting approximately 1 hour. The Center for Weight and Health at UC Berkeley processed data from the questionnaires.

Statistical analyses. For each of the research questions, the dependent variables were child-feeding practices and portion-size perceptions. Based on established methods (Carlson et al. 1999), responses to the USDA Food Security/ Hunger Core Module were dichotomized, and a participant responding affirmatively ("yes," "often" or "sometimes") to fewer than three of 18 questions was categorized as "food secure," while those responding affirmatively to three or more were categorized as "food insecure." To assess past food insecurity, participants who gave three or more positive responses to the 10 -item Past Food Insecurity Questionnaire 
were categorized as "past food insecure," and those responding affirmatively to fewer than three were categorized as "not past food insecure."

To examine the relationship between child-feeding practices, past and current maternal food insecurity, and child's weight, Wilcoxon Rank Sum tests were used to test these associations among most of the variables. For variables that were dichotomous, chisquare tests were used. Chi-square analyses were used to evaluate the relationship among pairs of the following categorical variables: dichotomous children's BMI, mother's BMI, and past and current food insecurity.

\section{Relationships and trends}

Mother's demographics. Most of the 123 participating mothers were recent immigrants from Mexico: 118 were born and 112 grew up there. Only eight grew up in the United States, and two had missing data. Ninety percent of the mothers reported speaking Spanish at home. The mothers' ages ranged from 20 to 50 years, with a median age of 29 years. Forty-three percent of the participants had 8 or fewer years of education, while the remaining $57 \%$ had from 9 to 12 years. Nearly all (94\%) reported monthly household incomes of $\$ 2,000$ or less.

Children's weights. Twenty-seven percent $(n=20)$ of the children in the sample were overweight, with BMIs more than or equal to the 95th percentile for children their age, and there was a strong relationship between mother's BMI and children's BMI (table 1). Mothers with BMIs of 30 or higher were more than twice as likely to have children with BMIs above the 95th percentile than mothers with BMIs below 30 (43\% versus 16\%, respectively) $(P<0.005)$.

Interestingly, less than one-third of the mothers with overweight children perceived their child as being overweight or heavier than children the same age. About half of the mothers with overweight children reported that a doctor or other health professional had told them their child was overweight. Sixty-four percent of the moth-

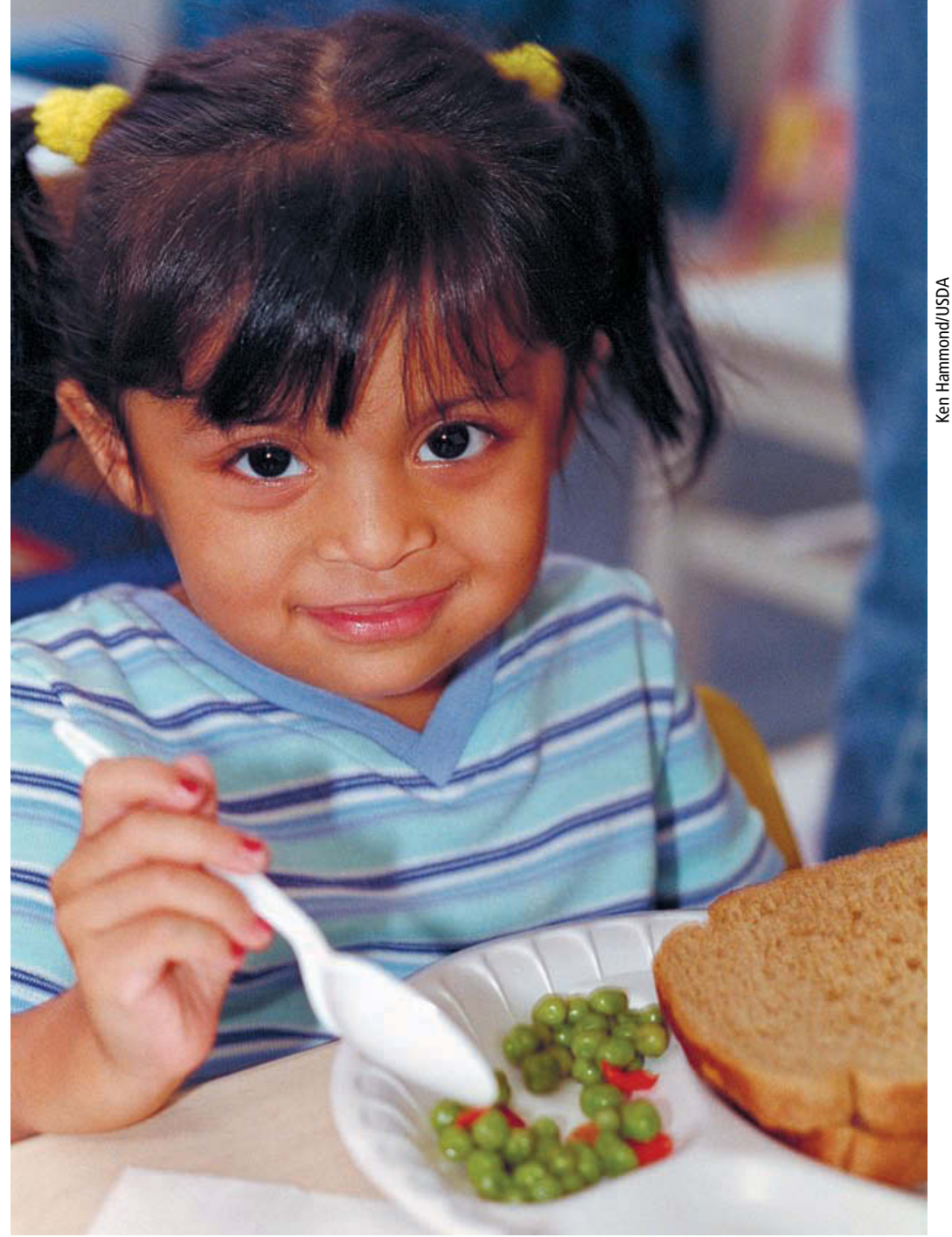

In the study, mothers who were currently food insecure were about twice as likely as those who were food secure to worry about their children overeating. Above, a girl eats lunch at a child-care center.

ers with overweight children were not concerned about their child becoming overweight, and $77 \%$ were not worried about their child eating too much food.

Food security and child weight. Over half $(n=66)$ of the 123 participants interviewed had experienced some form of past food insecurity; less than onequarter $(n=26)$ reported current food insecurity (table 2). Fourteen percent $(n=17)$ of the participants reported experiencing both past and current food insecurity. While not statistically significant, there appeared to be a trend for those who experienced food insecurity as a child to report current food insecurity $(P=0.18)$.

Of the 74 mothers who were weighed and measured, the mother's weight was not associated with their level of current food security. Thirty-one percent of those who had experienced past food insecurity were obese (BMI higher than 30 ), versus $53 \%$ of those who had not

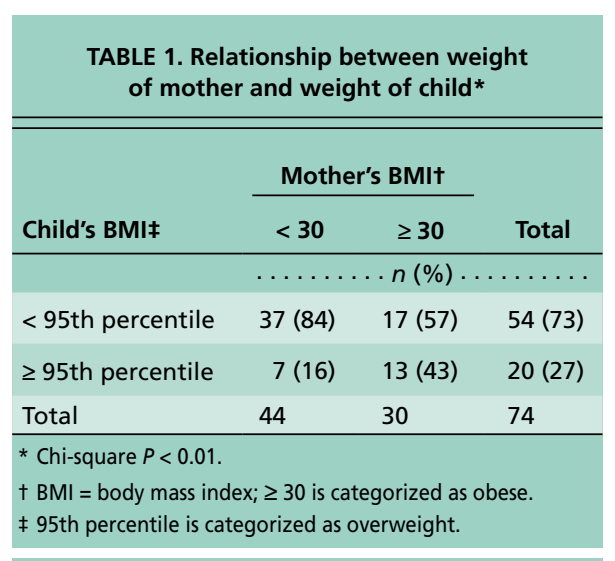

TABLE 2. Food-security status of all mothers, past and present $(n=123)$

\begin{tabular}{lccc}
\hline \hline & \multicolumn{2}{c}{ Past } & \\
\cline { 2 - 3 } Current & $\begin{array}{c}\text { Food } \\
\text { secure }\end{array}$ & $\begin{array}{c}\text { Food } \\
\text { insecure }\end{array}$ & Total \\
\hline Food secure & 48 & 49 & 97 \\
Food insecure & 9 & 17 & 26 \\
Total & 57 & 66 & 123 \\
\hline
\end{tabular}


experienced past food insecurity $(P=$ 0.054) (data not shown). There was no association between current or past maternal food security or insecurity and their child's weight.

Child feeding and mother's past food insecurity. Although not reaching statistical significance in this small study, a few trends seemed worth noting. Mothers who are currently food insecure may be less likely to keep track of the sweets their children eat $(P=0.069)$, and less likely to tell their children that they could not go out to play or get up to watch television until they eat $(P=$ 0.089 ). In addition, food-insecure mothers may be more likely to offer their children their favorite foods as a reward for good behavior $(P=0.088)$. Mothers who were food-insecure in the past may be less likely to believe that their children should always eat all of the food on their plate $(P=0.079)$.

Food security and child portion sizes. Mothers currently experiencing food insecurity seemed to offer their children smaller portions of certain foods such as French fries $(P<0.10)$ and chicken nuggets $(P<0.05)$ (table 3). On the other hand, mothers who experienced past food insecurity offered their children larger portions of orange juice $(P<0.05)$ and smaller portions of corn $(P<0.10)$ (Note: $P$ values below 0.05 are considered statistically significant; $P$ values below 0.10 are considered a statistical trend.)

Child-feeding practices and weight. Child-feeding style did not appear to be associated with mother's weight. The only parental feeding behavior significantly associated with BMI in children was that of mothers of overweight children, who were more likely than the other mothers to report scolding their child for not eating well $(P<0.05)$.

Concern over weight and food. Mothers who were currently food-insecure were about twice as likely as those who were food-secure to worry that their child eats too much food ( $42 \%$ versus $18 \%$, respectively) $(\mathrm{P}<0.01)$. About four times as many food-insecure mothers reported having been told by a physician or health professional that their child was overweight compared to mothers who are currently food

TABLE 3. Relationship between some portion sizes and current and past maternal food insecurity

\begin{tabular}{|c|c|c|}
\hline \multirow[b]{2}{*}{ Food portion (range of sizes) } & \multicolumn{2}{|c|}{ Food insecurity } \\
\hline & Current & Past \\
\hline \multicolumn{3}{|c|}{$\ldots \ldots \ldots \ldots$ P value (direction)* } \\
\hline Orange juice ( $1 / 2$ to $11 / 4$ cup) & $0.691(-)$ & $0.002+(+)$ \\
\hline Corn ( $1 / 4$ to 1 cup) & $0.155(-)$ & $0.096(-)$ \\
\hline French fries (38 to 101 grams) & $0.085(-)$ & $0.422(-)$ \\
\hline Chicken nuggets ( 45 to 150 grams, or 3 to 10 pieces) & $0.045+(-)$ & $0.184(-)$ \\
\hline \multicolumn{3}{|l|}{$\begin{array}{l}\text { * Sign in parenthesis indicates direction of association, with a minus } \\
\text { indicating smaller portions selected by food-insecure mothers. }\end{array}$} \\
\hline
\end{tabular}

secure (27\% versus $6 \%$, respectively). Forty-three percent of the children of food-insecure mothers were classified as overweight (BMI greater than the 95th percentile). As expected, mothers of overweight children were more likely to report being concerned that their child eats too much $(P=0.029)$. Accordingly, these mothers were much more likely to be concerned about child overweight $(\mathrm{P}<0.001)$.

\section{Addressing childhood overweight}

There were $60 \%$ more overweight children in our study population $(27 \%)$ than found in other national studies for low-income Latino children (17\%) (Polhamus et al. 2004). However, by definition our convenience sample of WIC-eligible urban and rural mothers of 3-to-5-year-old Latino children from two California counties was not representative.

Among Latino immigrants such as the young Mexican-American mothers in our study, acculturation to U.S. culture is associated with poor dietary habits, less physical activity and high rates of obesity (Ravussin et al. 1994; Unger et al. 2004). Subsequent generations of Latinos in the United States tend to increase their consumption of fast food, convenience foods, salty snacks, simple sugars, chocolate candy, and total added and saturated fats (Ayala et al. 2005; Neuhouser et al. 2004).

Because children's eating habits are largely developed and maintained in the home, parental feeding practices are of particular concern. While not statistically significant, mothers who were experiencing food insecurity tended to be less likely to use restrictive child-feeding practices and more likely to use food as a reward. At the same time, these mothers were more likely to worry that their child was eating too much food $(P<0.01)$. Accordingly, these mothers tended to be more likely to offer smaller portion sizes to their children. Others have reported that current food insecurity may affect the variety of foods available and consumed by families (Kaiser et al. 2003). It seems natural for mothers to limit foods in times of food insecurity, and to offer more when food is available. Our observations indicate that current food insecurity, as compared to past food insecurity, is more associated with child-feeding practices and weight status.

In our sample, we found few parenting differences between mothers with past or current food-insecurity. However, our study was limited by the small sample size. Even so, we did find trends among mothers with past food insecurity that suggest possible differences in factors associated with obesity. For example, past food-insecure mothers were less likely to be obese than mothers who had not experienced food insecurity in their childhoods $(P=0.054)$. In contrast to other studies showing a significant association between food insecurity and obesity in Latino mothers (Crawford, Gosliner, et al. 2004), we found that mothers who were currently food-insecure were no more likely to be obese than currently food-secure mothers.

The failure of many mothers to recognize that their children were overweight is an important finding. Studies have shown that Latino mothers associate thinness with poor health and being prone to disease 
$\checkmark$ UC nutrition educator Dolores Vallejo (left) and Angie Tazio, nutrition education program manager, demonstrate how they conducted the portion-size part of the study, which found that current food insecurity was more closely associated with child-feeding practices than past food insecurity.

(Crawford, Gosliner, et al. 2004). Other studies of food insecurity among Latino immigrants have confirmed the mismatch between excess weight in children and maternal concern. WIC mothers have been observed to disagree when educators identify their children as overweight (Chamberlin et al. 2002). It is possible that in a setting where such a high percentage of children are overweight, mothers may assume that higher weight levels are the norm. Furthermore, mothers may be reluctant to label or believe that their children are overweight (Myers and Vargas 2000). Finally, Latino mothers have suggested that their child will "grow out of it" or is genetically destined to be large (Crawford, Gosliner, et al. 2004).

This is the first study to examine associations between current and past food insecurity and child-feeding practices. The importance of studying this population is immense, due to the greater incidence of overweight and diabetes in low-income Latino children. While the issue of child overweight has received much attention, this UC Cooperative Extension study can help to elucidate appropriate intervention strategies, laying a foundation for teaching parents of preschoolers about the relationships between childhood obesity and child-feeding practices.

P.B. Crawford is Adjunct Professor, Cooperative Extension Specialist and Co-Director, Center for Weight and Health, UC Berkeley; C.L. Lamp is Nutrition, Family and Consumer Sciences Advisor, UC Cooperative Extension (UCCE), Tulare County; $Y$. Nicholson is Nutrition, Family and Consumer Sciences Advisor, UCCE Sacramento County; S. Krathwohl is Project Manager, Kaiser Permanente Division of Research, Oakland; M. Hudes is Senior Statistician, Center for Weight and Health UC Berkeley; and M.S. Townsend is Cooperative Extension Specialist, Department of Nutrition, UC Davis. The authors gratefully acknowledge the assistance of UC Berkeley students Elizabeth Okito and Robin Dean.

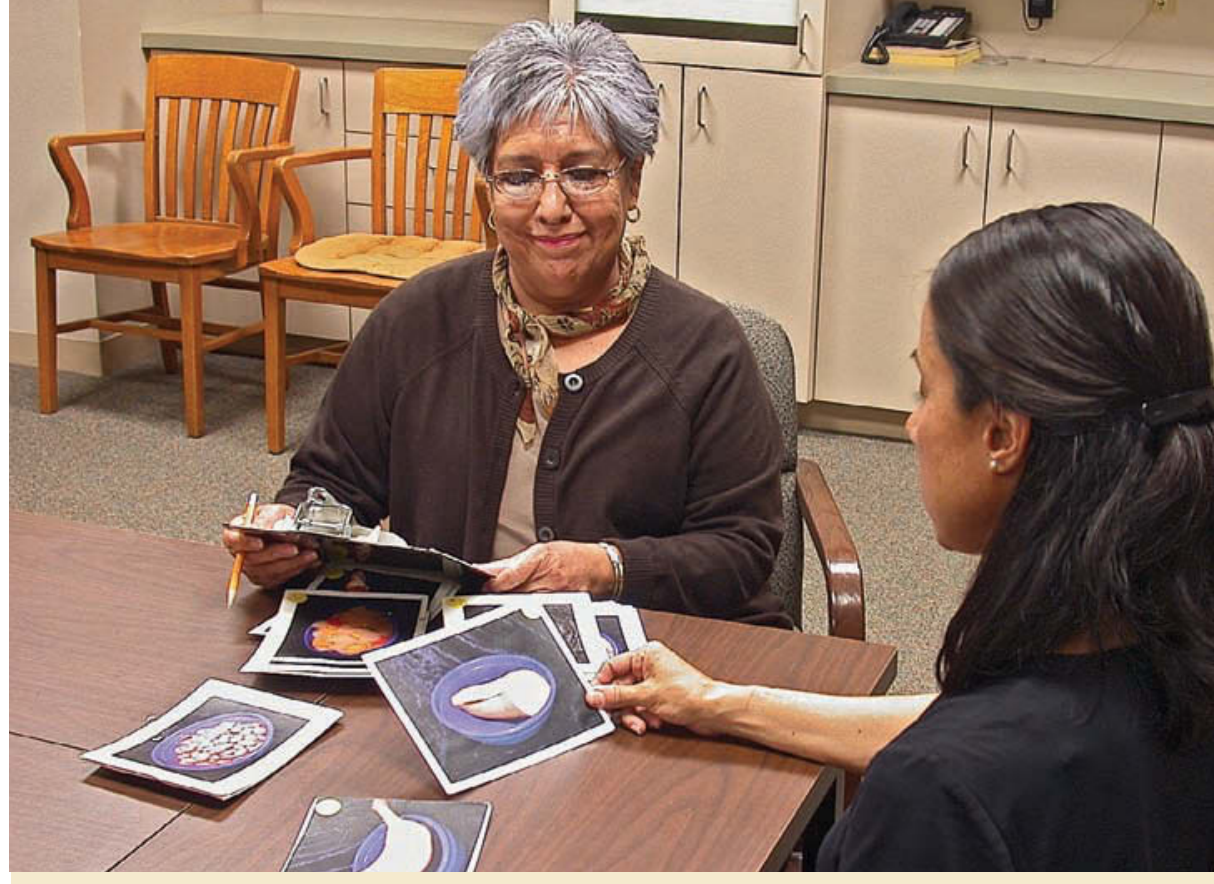

\section{References}

Ayala GX, Mueller K, Lopez-Madurga E, et al. 2005. Restaurant and food shopping selections among Latino women in Southern California. J Am Diet Assoc 105(1):38-45.

Bickel G, Nord M, Price C, et al. 2000. Guide to Measuring Household Food Security, Revised 2000. US Department of Agriculture, Food and Nutrition Service. Alexandria, VA.

Birch LL, Fisher JO, Grimm-Thomas K, et al. 2001 Confirmatory factor analysis of the Child Feeding Questionnaire: A measure of parental attitudes, beliefs and practices about child feeding and obesity proneness. Appetite 36:201-10.

Brewis A. 2003. Biocultural aspects of obesity in young Mexican school children. Am J Hum Bio $15: 446-60$

Carlson JC, Andrews SA, Bickel GW. 1999. Measuring food insecurity and hunger in the United States: Development of a national benchmark measure and prevalence estimates. J Nutr 129(2S Suppl):510-6S.

Chamberlin LA, Sherman SN, Jain A, et al. 2002 The challenge of preventing and treating obesity in low-income, preschool children: Perceptions of WIC health care professionals. Arch Pediatr Adolesc Med 156:662-8.

Crawford PB, Gosliner W, Anderson C, et al. 2004. Counseling Latina mothers of preschool children about weight issues: Suggestions for a new framework. Am Diet Assoc 104(3):387-94.

Crawford, PB, Story M, Wang MC, et al. 2001. Ethnic issues in the epidemiology of child obesity. Ped Clinic North Am 48(4):855-79.

Crawford PB, Townsend M, Metz D, et al. 2004 How can Californians be overweight and hungry? Cal Ag 58 (1):12-7.

Engell D, Kramer M, Zaring D, et al. 1995. Effects of serving size on food intake in children and adults. Obes Res 3 (Suppl 3):381S.

Fisher JO, Birch LL. 1999. Restricting access to foods and child's eating. Appetite 32:405-19.

Johnson SL. 2000. Improving preschoolers' selfregulation of energy intake. Pediatrics 106:1429-35

Kaiser L, Melgar-Quiñonez H, Townsend MS, et al. 2003. Food insecurity and food supplies in Latino households with young children. J Nutr Educ Behav 35:148-53.

Kempson KM, Keenan DP, Sadani PS, et al. 2002. Food management practices used by people with limited resources to maintain food sufficiency as reported by nutrition educators. J Am Diet Assoc 102:1795-9.
Kendall A, Olson CM, Frongillo EA Jr. 1996. Relationship of hunger and food insecurity to food availability and consumption. J Am Diet Assoc 96:1019-24.

McConahy KL, Smiciklas-Wright $\mathrm{H}$, Birch LL, et al. 2002. Food portions are positively related to energy intake and body weight in early childhood. J Pediatr 140:340-7.

Myers S, Vargas Z. 2000. Parental perceptions of the pre-school obese child. Pediatr Nurs 26:23-30.

[NCHS] National Center for Health Statistics. 2004 Prevalence of Overweight Among Children and Adolescents: United States, 1999-2002. www.cdc.gov/nchs/ products/pubs/pubd/hestats/overwght99.htm (accessed Feb. 2005).

Neuhouser ML, Thompson B, Coronado GD, Solomon CC. 2004. Higher fat intake and lower fruit and vegetables intakes are associated with greater acculturation among Mexicans living in Washington State. J Am Diet Assoc 104(1):51-7

Nord M, Andrews M, Carison S. 2003. Household Food Security in the United States, 2002. Food and Rura Economics Division, Economic Research Service, US Department of Agriculture. Food Assistance and Nutrition Research Rep No 35

Olson CM. 1999. Nutrition and health outcomes associated with food insecurity and hunger. J Nut 129:521-4S

Polhamus B, Dalenius K, Thompson D, et al. 2004. Pediatric Nutrition Surveillance 2003 Report. US Dept of Health and Human Services, Centers for Disease Control and Prevention. Atlanta, GA.

Ravussin E, Valencia ME, Esparza J, et al. 1994. Effects of a traditional lifestyle on obesity in Pima Indians. Diabetes Care 17(9):1067-74.

Ribar DC, Hamrick KS. 2003. Dynamics of Poverty and Food Sufficiency. Food and Rural Economics Division, Economic Research Service, US Department of Agriculture. Food Assistance and Nutrition Research Rep No 36

Rolls BJ, Morris EL, Roe LS. 2002. Portion size of food affects energy intake in normal-weight and overweight men and women. Am J Clin Nutr 76:1207-13.

Townsend MS, Peerson J, Love B, et al. 2001. Food insecurity is positively related to overweight in women. J Nutr 131:1738-45.

Unger JB, Reynolds K, Shakib S, et al. 2004. Acculturation, physical activity, and fast-food consumption among Asian-American and Hispanic adolescents. J Community Health 29(6):467-81

[USDA] US Department of Agriculture. 1999. Tips for Using the Food Guide Pyramid for Young Children 2 to 6 Years Old. Center for Nutrition Policy and Promotion. wuw.mypyramid.gov/index.html (accessed May 17, 2005) 